Revue musicale OICRM

\title{
La Revue musicale ou le phoenix musical
}

\section{Michel Duchesneau}

Volume 4, numéro 2, 2017

Enjeux culturels dans la presse musicale française, 1900-1925

URI : https://id.erudit.org/iderudit/1043218ar

DOI : https://doi.org/10.7202/1043218ar

Aller au sommaire du numéro

\section{Éditeur(s)}

Observatoire interdisciplinaire de création et recherche en musique (OICRM)

ISSN

2368-7061 (numérique)

Découvrir la revue

\section{Citer cet article}

Duchesneau, M. (2017). La Revue musicale ou le phoenix musical. Revue musicale OICRM, 4(2), 19-34. https://doi.org/10.7202/1043218ar

\section{Résumé de l'article}

Lorsque le directeur de La Revue musicale sIM, Jules Écorcheville, part pour le front en 1914, il écrit à son ami Émile Vuillermoz :

Si je ne reviens pas, je vous recommande notre oeuvre, cher ami. Et surtout, si vous tenez à me faire plaisir dans l'autre monde, efforcez-vous de maintenir la concorde et l'harmonie entre les différents éléments qui vont se trouver en présence à ma disparition. Notre revue est faite de différentes pièces ajustées (Amis, sıM, etc.), qui tiennent en équilibre par miracle, quelques années de cohésion sont absolument nécessaires encore et c'est précisément cette concentration de nos différentes forces qu'il faudrait maintenir. En tout cas, il ne faudrait pas que ma disparition entraînât celle d'une oeuvre qui nous a coûté, à tous, tant de peine. N'est-il pas vrai ?

Malgré le souhait d'Écorcheville, La Revue sıM disparaîtra, mais pas pour longtemps puisqu'elle donnera naissance à deux nouveaux organismes en 1917 et 1920 . Pendant la guerre, les anciens de La Revue sIM dont Lionel de La Laurencie, vont créer la Société française de musicologie (SFM) sur les ruines de la Société internationale de musique et publieront un Bulletin qui deviendra la Revue de musicologie. Loin de l'actualité, s'écartant délibérément du contexte sociopolitique et culturel, la SFM et son Bulletin favoriseront une approche très " scientifique " et relativement nouvelle en France, de la musicologie, bien qu'encore teintée par les tendances historicisantes à la manière de la Schola Cantorum et écartant pour un temps toute la musicologie germanique. En parallèle, sous les auspices du musicologue Henry Prunières qui s'écarte résolument de la SFM, est créée La Revue musicale. C'est à partir d'un réseau international qui place la musicologie française au coeur de l'action musicale contemporaine que Prunières établit de nouvelles alliances avec le milieu des arts et de la littérature pour fonder l'une des plus célèbres revues musicales de la première moitié $d u \mathrm{xx}^{\mathrm{e}}$ siècle. À partir de documents inédits, nous étudierons les circonstances qui mènent à la refondation de La Revue musicale sur les cendres de la Revue sim entre 1915 et 1919. Nous verrons ainsi comment les hasards de la guerre mènent Prunières à entreprendre la carrière d'éditeur et comment le musicologue conçoit le projet international de la revue dans un contexte de guerre qui contribue à une redéfinition des cultures nationales.

Il est difficile d'extrapoler sur ce qu'aurait pu être l'avenir de La Revue musicale SIM si la guerre n'avait pas eu lieu. Il est par contre possible de documenter et de comprendre le rôle que la Grande Guerre jouera dans l'essor d'une nouvelle dynamique pour la musicologie française dont la division d'abord justifiée par le conflit aura des conséquences à long terme sur l'échiquier international de la discipline.
Ce document est protégé par la loi sur le droit d'auteur. L'utilisation des services d'Érudit (y compris la reproduction) est assujettie à sa politique d'utilisation que vous pouvez consulter en ligne.

https://apropos.erudit.org/fr/usagers/politique-dutilisation/ 


\title{
La Revue musicale ou le phoenix musical
}

\author{
Michel Duchesneau
}

\section{Résumé}

Lorsque le directeur de La Revue musicale SIM, Jules Écorcheville, part pour le front en 1914, il écrit à son ami Émile Vuillermoz :

Si je ne reviens pas, je vous recommande notre œuvre, cher ami. Et surtout, si vous tenez à me faire plaisir dans l'autre monde, efforcez-vous de maintenir la concorde et l'harmonie entre les différents éléments qui vont se trouver en présence à ma disparition. Notre revue est faite de différentes pièces ajustées (Amis, sIM, etc.), qui tiennent en équilibre par miracle, quelques années de cohésion sont absolument nécessaires encore et c'est précisément cette concentration de nos différentes forces qu'il faudrait maintenir. En tout cas, il ne faudrait pas que ma disparition entraînât celle d'une œuvre qui nous a coûté, à tous, tant de peine. N'est-il pas vrai?

Malgré le souhait d'Écorcheville, La Revue SIM disparaîtra, mais pas pour longtemps puisqu'elle donnera naissance à deux nouveaux organismes en 1917 et 1920. Pendant la guerre, les anciens de La Revue sIM dont Lionel de La Laurencie, vont créer la Société française de musicologie (SFM) sur les ruines de la Société internationale de musique et publieront un Bulletin qui deviendra la Revue de musicologie. Loin de l'actualité, s'écartant délibérément du contexte sociopolitique et culturel, la SFM et son Bulletin favoriseront une approche très "scientifique » et relativement nouvelle en France, de la musicologie, bien qu'encore teintée par les tendances historicisantes à la manière de la Schola Cantorum et écartant pour un temps toute la musicologie germanique. En parallèle, sous les auspices du musicologue Henry Prunières qui s'écarte résolument de la SFM, est créée La Revue musicale. C'est à partir d'un réseau international qui place la musicologie française au cœur de l'action musicale contemporaine que Prunières établit de nouvelles alliances avec le milieu des arts et de la littérature pour fonder l'une des plus célèbres revues musicales de la première moitié $d u x^{e}$ siècle. À partir de documents inédits, nous étudierons les circonstances qui mènent à la refondation de La Revue musicale sur les cendres de la Revue SIM entre 1915 et 1919. Nous verrons ainsi comment les hasards de la guerre mènent Prunières à entreprendre la carrière d'éditeur et comment le musicologue conçoit le projet international de la revue dans un contexte de guerre qui contribue à une redéfinition des cultures nationales.

Il est difficile d'extrapoler sur ce qu'aurait pu être l'avenir de La Revue musicale SIM si la guerre n'avait pas eu lieu. Il est par contre possible de documenter et de comprendre le rôle que la Grande Guerre jouera dans l'essor d'une nouvelle dynamique pour la musicologie française dont la division d'abord justifiée par le conflit aura des conséquences à long terme sur l'échiquier international de la discipline.

Mots clés : Revue musicale ; musicologie ; Écorcheville ; Prunières ; Guerre 14-18 ; presse musicale. 


\begin{abstract}
When the director of La Revue SIM, Jules Ecorcheville, left for the front in 1914, he wrote to his friend Émile Vuillermoz:

If I do not come back, I recommend our work, dear friend. And above all, if you want to please me in the other world, try to maintain concord and harmony between the various elements that will be in the presence of my disappearance. Our periodical is made of different adjusted pieces (Friends, SIM, etc.), which balance by miracle, a few years of cohesion are absolutely necessary again and it is precisely this concentration of our different strengths that should be maintained. In any case, my disappearance should not entailed that of a work which cost us, all, so much trouble. Is not it true?
\end{abstract}

Despite the wish of Écorcheville, La Revue SIM will disappear, but not for long since it will give birth to two new organizations in 1917 and 1920. During the war, the alumni of La Revue SIM including Lionel de La Laurencie, will create the Company French musicology (SFM) on the ruins of the Sociéte internationale de musique and will publish a Bulletin that will become the Revue de musicologie. Far from the current events, deliberately departing from the socio-political and cultural context, the SFM and its Bulletin will favor a very "scientific" and relatively new approach in France, of musicology, although still tinged by the historicizing trends in the manner of the Schola Cantorum and dismissing for a time all Germanic musicology. In parallel, under the auspices of the musicologist Henry Prunières who resolutely deviates from the SFM, is created La Revue musicale. It is from an international network that places French musicology at the heart of contemporary musical action that Prunières establishes new alliances with the world of arts and literature to found one of the most famous musical journals of the world of the first half of the $20^{\text {th }}$ century. From unpublished documents, we will study the circumstances that lead to the re-founding of La Revue musicale on the ashes of the Revue SIM between 1915 and 1919. We will thus see how the chances of war lead Prunières to undertake the publishing career and how the musicologist conceives the international project of the journal in a context of war that contributes to a redefinition of national cultures.

It is difficult to extrapolate what the future of La Revue SIM would have been if the war had not taken place. On the other hand, it is possible to document and understand the role that the Great War will play in the development of a new dynamic for French musicology whose division first justified by the conflict will have long-term consequences on the chessboard. international discipline.

Keywords: Revue musicale; musicology; Écorcheville; Prunières; World War One; musical press.

L'entrée en guerre de la France bouleverse l'activité quotidienne du pays et le milieu de la presse musicale est évidemment profondément perturbé. Le 5 septembre 1914, la rédaction du Ménestrel publie un avis qui résume parfaitement la situation :

Comme nous l'avions promis à nos abonnés et à nos lecteurs, nous avons tout fait pour paraître le plus longtemps possible. Mais aujourd'hui, que, par suite de départs, nos rangs s'éclaircissent de plus en plus, que les nouvelles artistiques deviennent de plus en plus rares et que leur intérêt, devant le drame qui se développe si tragiquement en Europe, est vraiment médiocre, nous nous voyons, à notre grand regret, contraints de suspendre la publication du Ménestrel (La direction 1914, p. 273). 
Certaines revues musicales comme Musica et La Revue musicale SIM, dont le dernier numéro paraît en juillet $1914^{1}$, disparaîtront carrément.
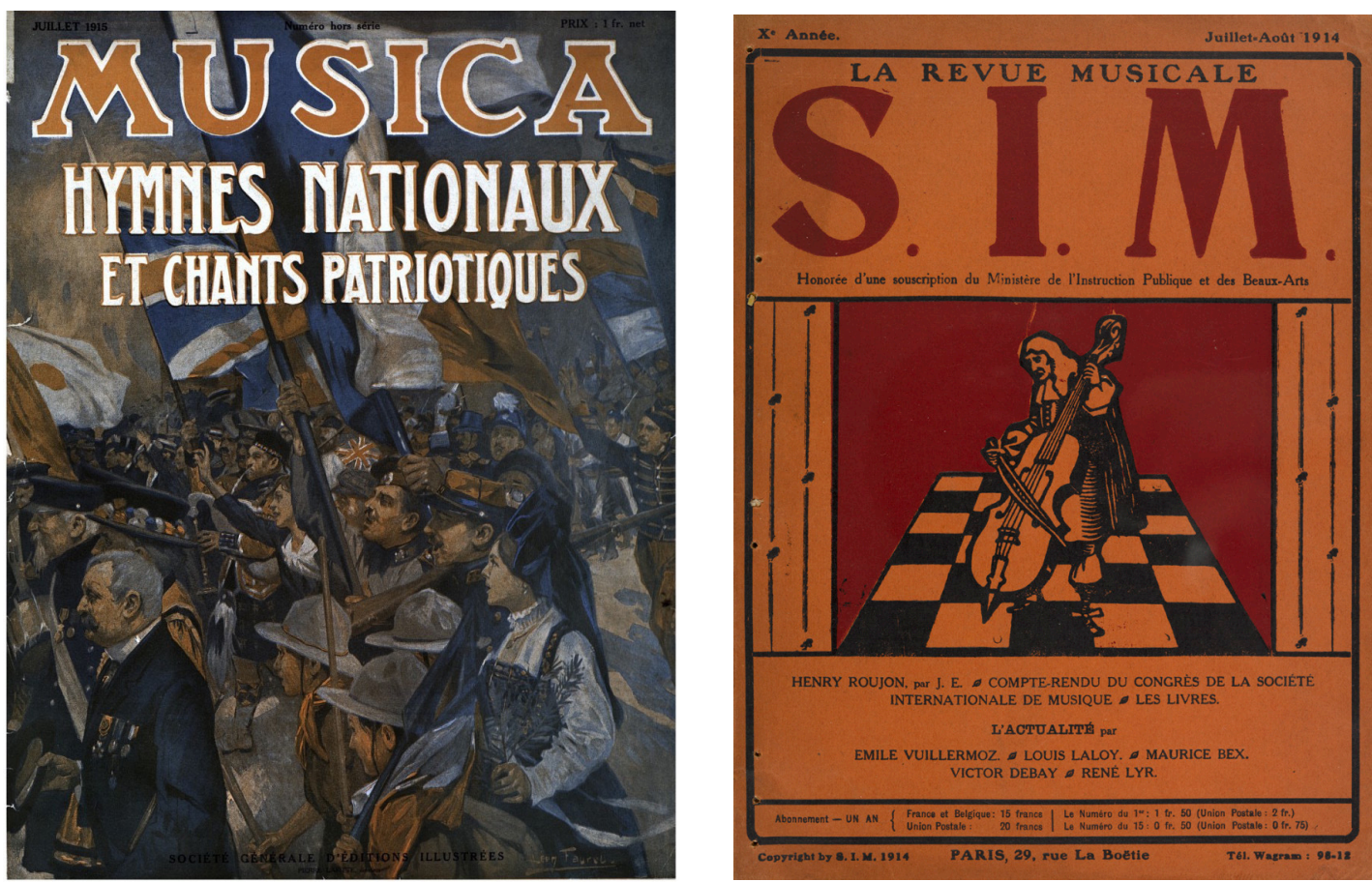

Figure 1: Pages de couverture de Musica de 1917 et de La Revue musicale SIM de 1914.

D'autres, par contre, reprendront progressivement leurs activités. Ce sera le cas du Courrier musical qui, après avoir été fusionné à la Revue sIM en 1913, reprendra ses activités autonomes sporadiquement de 1916 à 1918 puis régulièrement à partir de 1919. Le cas de la disparition de la Revue SIM est intéressant, car en 1920, telle un phœnix, elle va renaître de ses cendres sous le nom de Revue musicale sur des bases internationalistes qui témoignent d'un engagement ferme de la part de son refondateur, le musicologue Henry Prunières. Cette renaissance s'accompagne de transformations importantes dues, en bonne partie, aux circonstances de la Grande Guerre, mais aussi au capital culturel et économique de Prunières qui saura en user avec habileté. C'est cette destinée singulière qui fera l'objet du présent article.

Notre objectif est donc de reconstituer pas à pas, les origines de La Revue musicale en identifiant, entre autres, les principaux artisans (administrateurs, donateurs et collaborateurs) qui participeront à la résurrection de la revue et qui progressivement intégreront le réseau développé par Prunières pendant la guerre. Comme nous tenterons de le démontrer au fil de cet article, la rupture provoquée par le conflit et qui se traduit par la fin des activités de la Revue SIM. ne sera que partielle puisque la nouvelle revue reprendra à son compte la majorité des orientations éditoriales de la 
Revue SIM, l'essentiel de sa structure éditoriale, et s'appuiera sur un certain nombre de collaborateurs qui œuvraient auparavant pour la Revue SIM.

Nous formulons l'hypothèse que s'intéresser à l'origine de La Revue musicale d'un point de vue historique apporte une série de données fondamentales pour la compréhension de son fonctionnement, mais aussi de son orientation musicologique, voire esthétique lorsqu'il s'agit de défendre la musique ancienne ou encore les courants d'avant-garde français et étrangers. Les racines italiennes du réseau de Prunières permettent ainsi de comprendre pourquoi les compositeurs italiens comme Gian Francesco Malipiero et Alfredo Casella occuperont une place privilégiée dans les pages de la revue alors que l'histoire en a jugé autrement.

\section{LA REVUE MUSICALE SIM}

La revue est le résultat de fusions et de transformations successives documentées dans d'autres travaux ${ }^{2}$ que je simplifierai ici en une courte présentation illustrée par un tableau :

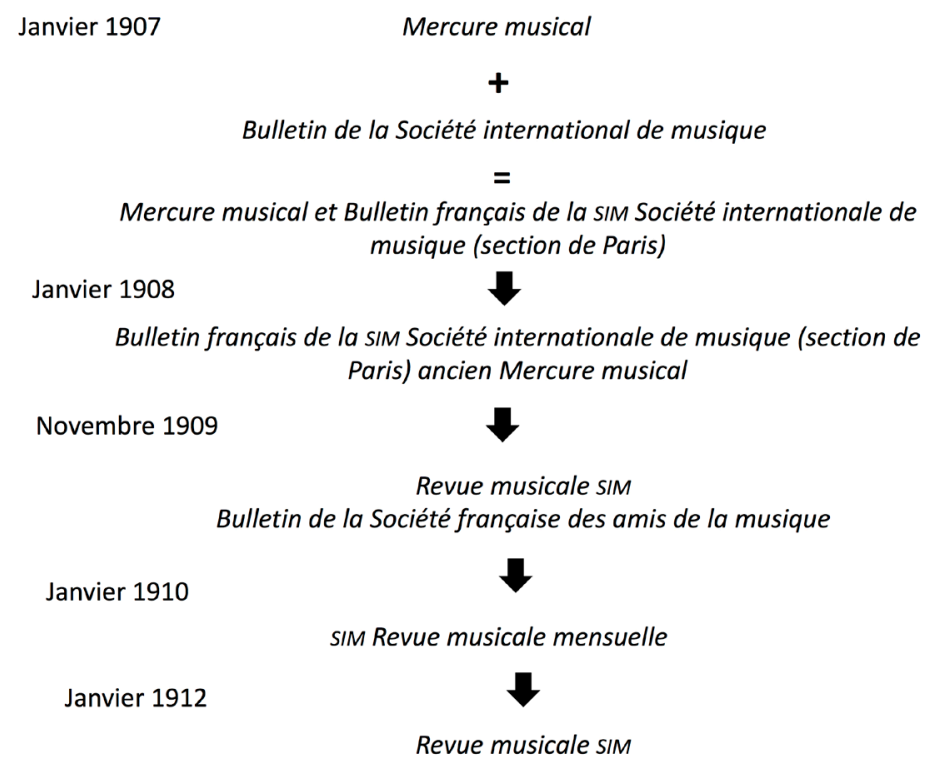

Figure 2 : Généalogie de La Revue musicale sim.

En 1907, le musicologue Jules Écorcheville fait du Mercure musical fondé en 1905 par Louis Laloy et Jean Marnold, le Bulletin de la section française de la Société internationale de musique. Sous son impulsion, la musicologie française s'inscrit désormais dans une dynamique internationale. Aux yeux de ses artisans, elle est " en France la seule revue de musique capable de publier des articles étendus, et comptant déjà parmi ses collaborateurs attitrés la plus grande partie des membres français de la société internationale » ("Avis aux lecteurs » 1907). La revue est rapidement investie par la SIM :

2 Pour des détails concernant l'histoire de la Revue SIM avant la guerre voir, entre autres, Segond-Genovesi 2015 et Duchesneau 2015. 
le numéro du 15 février 1907 comporte en dernière partie une section entièrement consacrée à la SIM où sont consignés son histoire, la liste des publications, les statuts généraux de la SIM, une liste des membres actifs de la section de Paris et les statuts spécifiques à la section parisienne.

En janvier 1908, la revue devient le Bulletin français de la SIM Société internationale de musique (section de Paris), ancien Mercure musical. En 1909, la revue joue un rôle supplémentaire et devient aussi le Bulletin de la Société française des amis de la musique (SFAM) dont on retrouve les documents constitutifs dans les premières pages du numéro de juillet 1909. En 1912, la revue d'Écorcheville absorbe La Revue musicale fondée en 1902 par Jules Combarieu et, en 1913, elle engloutit Le Courrier musical. Ces fusions et collaborations font de La Revue musicale SIM une revue au lectorat nombreux, tant en France qu'à l'étranger, où musicologues, compositeurs et critiques musicaux les plus en vue de l'époque écrivent articles, essais et critiques sous la gouverne du musicologue Jules Écorcheville. Ce dernier déploie une grande énergie à maintenir en un tout cohérent, tant de collaborateurs venus de tous les horizons artistiques et politiques, tout comme il travaille avec acharnement à conserver un lectorat nombreux au sein des milieux musicologiques, artistiques et intellectuels.

\section{LA GUERRE}

Lorsque Jules Écorcheville part pour le front en 1914, il écrit à son ami Émile Vuillermoz, rédacteur en chef de la revue :

Si je ne reviens pas, je vous recommande notre œuvre, cher ami. Et surtout, si vous tenez à me faire plaisir dans l'autre monde, efforcez-vous de maintenir la concorde et l'harmonie entre les différents éléments qui vont se trouver en présence à ma disparition. Notre revue est faite de différentes pièces ajustées (Amis, sIM, etc.), qui tiennent en équilibre par miracle, quelques années de cohésion sont absolument nécessaires encore et c'est précisément cette concentration de nos différentes forces qu'il faudrait maintenir. En tout cas, il ne faudrait pas que ma disparition entraînât celle d'une œuvre qui nous a coûté, à tous, tant de peine. N'est-il pas vrai ? (Écorcheville 1914) ${ }^{3}$.

En toute logique, le fondateur de la revue souhaite que son œuvre dure et qu'elle continue au lendemain du conflit. Dans les faits, la correspondance du musicologue avec sa femme nous révèle une situation plus nuancée compte tenu du fait que la revue est étroitement associée à la SIM dont Écorcheville est alors le président. En janvier 1915, il écrit à sa femme :

J'ai une lettre de Laloy [...] Il est d'avis pour la sIM de ne rien décider ni faire avant la fin de la guerre. C'est le plus sage. Mais V (Vuillermoz) et D (Vincent d'Indy) la trouvent mauvaise, parce que la situation est précaire! Ainsi m'ont-ils écrit et je leur ai répondu très fermement que j'étais décidé à ne rien faire du tout pour le

3 Correspondance aimablement communiquée par Cédric Segond-Genovesi, comme d'ailleurs toutes les lettres tirées du Fonds Jules Écorcheville citées dans cet article. 
moment. Je suis même résolu à ne plus reprendre seul le fardeau de la revue. J'ai beaucoup réfléchi à tout cela, et je trouverai dans la guerre l'occasion que je cherche depuis plusieurs années de ne plus avoir dans la sim qu'un rôle musicologique tout en m'assurant un revenu fixe. Je céderai à une société la Revue et ma collaboration (limitée) contre une mensualité assurée ; et je continuerai ou reprendrai mes travaux d'érudition [...] Ce sont des projets qui supposent une contrepartie. Mais j'ai maintenant assez de gens dont la revue assure la situation pour que ceux-ci aient [un] intérêt majeur à trouver cette contrepartie. Le tout était d'avoir un prétexte plausible. Le présent cataclysme en est un (Écorcheville 1915a).

Écorcheville fait référence à l'orage qui gronde dans les milieux musicologiques français après le congrès de la sIM qui s'était tenu en juin 1914 à Paris. Pour plusieurs, dont Julien Tiersot et Lionel de La Laurencie, l'internationalisme de la SIM n'est qu'une façade et la musicologie est, dans les faits, dominée par les musicologues de langue allemande. La guerre offre une occasion idéale pour ces musicologues de concevoir un projet d'une société musicologique indépendante et française. La mort d'Écorcheville sur le champ de bataille en 1915 semble avoir indirectement facilité la tâche aux musicologues nationalistes pour créer la Société française de musicologie (SFM) sur les ruines de la Société internationale de musique en 1917. La Revue musicale SIM disparaît alors définitivement au profit du Bulletin de cette nouvelle société qui deviendra la Revue de musicologie. Loin de l'actualité, s'écartant délibérément du contexte sociopolitique et culturel, la SFM et son Bulletin favoriseront une approche très "scientifique» et relativement nouvelle en France de la musicologique, bien qu'encore teintée par les tendances historicisante à la manière de la Schola Cantorum et écartant pour un temps toute la musicologie germanique (Iglesias 2009, p. 208, 211 et suivantes).

Mais revenons à l'histoire d'Écorcheville. Même s'il insiste auprès de Vuillermoz et de Laloy sur l'importance de conserver la Revue SIM après la guerre, il est clair qu'il n'en sera plus le directeur. Le 24 janvier 1915, il confirme à sa femme que sa décision est prise de quitter la SIM :

Oui nous sommes bien d'accord. Nous l'étions d'ailleurs depuis longtemps sur la Revue, et dès 1909 mon intention était de la passer à d'autres. Mais il faut pour cela deux choses : $1^{\circ}$ un prétexte, $2^{\circ}$ un amateur.

Le prétexte sera la guerre ; celui-là est trouvé et bien trouvé. J'ai déjà annoncé mon intention à Doire 4 et à $\mathrm{V}$ [Vuillermoz] en leur disant que je désirais en rentrant ne pas reprendre le fardeau administratif de la Revue, et rester avec eux simplement pour que mon nom soit garant de la valeur rédactionnelle de la publication. J'ai ajouté qu'il faudrait faire une société anonyme, trouver des capitaux et que j'apporterais de mon côté le fruit de mes efforts depuis 8 ans.

Reste à réaliser la seconde partie de ce programme. Si la guerre dure encore quelque temps, et que les affaires reprennent, je compte sur Doire pour se mettre en campagne avant mon retour même et combiner quelque chose (Écorcheville 1915b).

4 René Doire (1879-1959), compositeur et chef d'orchestre, sera le rédacteur en chef au Courrier musical qui est absorbé par la revue d'Écorcheville en 1913. Doire est alors engagé comme administrateur de la revue. 
Le prétexte pour transformer la revue et surtout permettre à Écorcheville de s'en départir, sera donc la guerre. Mais en ce qui a trait à l'" amateur » de direction de revue musicale qui devra prendre l'affaire en main, il ne sera pas facile de le dénicher. Écorcheville avait-il en tête alors que Prunières serait cet " amateur » ? Ce n'est pas impossible, mais difficile à vérifier dans l'état actuel de nos recherches. D'après le témoignage de Prunières, les deux hommes étaient assez proches puisqu'en 1914, Écorcheville demande à Prunières d'assumer, " à la dernière minute " les fonctions de secrétaire du congrès de la SIM qui réunissait "plus de six cents congressistes » (Lefèvre 1929, p. 5). Prunières précise que c'est à cette occasion qu'il se liera d'amitié avec d'Annunzio.

Écorcheville meurt le 19 février 1915 alors qu'il dirige une attaque de ses soldats du $130^{\circ}$ régiment d'infanterie à Perthes-les-Hurlus dans 1'Oise. Avec sa disparition, pour ses contemporains, disparaît la Revue SIM. Vuillermoz écrivait en 1916 : «Il tomba glorieusement sans perdre la foi en son œuvre et sans croire compromis, par sa disparition, l'édifice qu'il avait si patiemment construit. Heureuse illusion, que ses amis ont eu la consolation de lui voir emporter dans la mort !»(Vuillermoz 1916, p. 34).

On peut se questionner sur le fait que Vuillermoz, aurait dû reprendre le flambeau puisqu'il était avec Écorcheville le principal moteur de la revue, mais il n'en fut rien. Dans une lettre à Madeleine Écorcheville de décembre 1915, il refuse dans un premier temps de contribuer à l'hommage en mémoire de Jules Écorcheville ne voulant pas se « voir substituer pour ce projet comme pour tous les autres, [aux] personnalités appelées à continuer l'œuvre de votre mari et à répondre aux espoirs qu'il formula dans ses derniers vœux »(Vuillermoz 1915). Il ajoute :

Je me ferais, d'ailleurs, un scrupule d'enlever au nouveau rédacteur en chef d'SIM [sic]. une occasion précieuse de justifier la confiance dont il est investi et je respecte trop les règles de la courtoisie confraternelle - même lorsqu'on s'en affranchit à mon égard - pour ne pas m'effacer avec empressement devant un successeur (ibid.).

Vuillermoz semble donc écarté de la succession pour la direction de la Revue SIM, ce qui n'est pas si étonnant lorsque l'on sait qu'il milite pour la disparition de la section française de la société internationale au profit d'une nouvelle société nationale. Mais dans les faits, il participera à la plaquette commémorative Le tombeau de Jules Écorcheville (Paris, Dorbon, 1916) avec Laloy et La Laurencie. Est-ce ce dernier qui doit prendre la place d'Écorcheville? Dans l'esprit d'Écorcheville, peut-être, mais comme nous l'avons déjà mentionné, La Laurencie, germanophobe et nationaliste, convainc les membres de dissoudre la section parisienne de la SIM pour fonder la Société française de musicologie en 1917 (Iglesias 2009, p. 205-206). Cette décision scelle définitivement le sort de la Revue sIM.

\section{UN NOUVEAU JOUEUR}

En août 1914, la guerre surprend Prunières à Lion-sur-mer alors qu'il passe des vacances en famille. Pour des raisons de santé, ayant le cœur très fragile, Prunières est affecté au "service auxiliaire » du rapatriement de la mairie du ve arrondissement : "Je dirige déjà un bureau, décidément, j’étais né administrateur ", écrit-il 
à sa femme (Prunières 1914a). Prunières se révèle alors un véritable patriote, pour lequel la défense de la Patrie est un devoir moral. Tout en rassurant sa femme dans sa correspondance journalière, il glorifie les faits d'armes tant français qu'anglais à travers les nouvelles reçues à Paris qui, il faut le reconnaître, sont à la fois autant d'informations que de désinformations. À témoin ces deux extraits de lettres où Prunières annonce à sa femme le débarquement de troupes cosaques puis japonaises pour sauver la France : "Coup de théâtre, mon chéri ! Le facteur dont parlait Lord Kitchener, c'est le débarquement incessant des Cosaques à Ostende. L'Écosse en est pleine ! Le tuyau est certain. M. Wilfrid Monod rentre d'Écosse et nous l'a dit à la mairie " (Prunières 1914b, $1^{\text {re }}$ lettre). Un peu plus tard dans la journée, démenti et nouvelle annonce :

On s'accorde à croire que " le nouveau et formidable facteur de succès qui étonnera l'Europe » dont parle Lord Kitchener est le débarquement prochain de troupes japonaises transportées clandestinement par les Anglais. Ce serait épatant ! (Prunières 1914b, 2e lettre).

$\mathrm{Au}$ début 1915, Prunières tombe malade. Hospitalisé au Val-de-Grâce, il sera transporté pour sa convalescence à l'hôpital Michelet où il passera de nombreuses semaines. En mars 1915, on lui demande d'organiser un concert pour les prisonniers de guerre. "Jusque-là, on n'avait entendu à Paris que des concerts de charité consistant en l'exhibition de $\mathrm{M}^{\text {lle }}$ Chenal $^{5}$ chantant La Marseillaise, à qui succédait Dranem ${ }^{6}$ puis Mistinguett » (Lefèvre 1929, p. 5) raconte Prunières qui organisa finalement un concert à la Salle Gaveau avec la participation de Ninon Vallin, Debussy, Georges Enesco, Ricardo Viñes, Henri Fabert et Alfredo Casella. Prunières réintègre les Services auxiliaires, mais est "mis en sursis ". À compter de cette époque, le musicologue travaille régulièrement avec Alfred Cortot qui est alors affecté au Service de la propagande du Sous-secrétaire des Beaux-Arts au Ministère de l'instruction publique et des Beaux-Arts pour l'organisation de manifestations musicales en France, mais aussi à l'étranger.

En mai 1916, Prunières fort de sa connaissance de l'Italie et de la langue du pays $^{7}$ est envoyé par le Ministère des Affaires étrangères en Italie. Il rejoint la mission économique dirigée par Paul Claudel qui est sur place depuis octobre $1915^{8}$.

\footnotetext{
5 Marthe Chenal (1881-1947), soprano dramatique, se produira très régulièrement l'Opéra Garnier et l'Opéra-comique. Pendant la guerre, elle chante La Marseillaise à la fin des représentations auxquelles elle participe à l'Opéra-Comique.

6 Il s'agit du nom d'artiste de Charles Armand Ménard (1869-1935), qui fit carrière comme chanteur humoriste et connut un vif succès dans le monde du Café-concert au tournant du $\mathrm{xx}^{\mathrm{e}}$ siècle.

$7 \quad$ Prunières connaît très bien l'Italie pour y avoir séjourné à plusieurs reprises. Son premier voyage remonte à 1910, alors qu'il entame des recherches dans les fonds d'archives du Vatican pour sa thèse de doctorat. Ce sera Romain Rolland, son directeur de recherche, qui lui ouvrira les portes du milieu musicologique et archivistique italien que ce dernier fréquente depuis longtemps. Voir à ce propos l'article de Catherine Massip, « Romain Rolland, maître, mentor et ami » (Massip 2015).

8 Dans son journal, Paul Claudel note : «Le 21 septembre, j’apprends ma nomination à Rome, chargé d'une mission économique en vue d'étudier la place que la rupture des relations avec l'Allemagne peut faire
} 
Prunières fait donc la connaissance du célèbre écrivain en arrivant à Rome dans des circonstances particulières, la guerre dominant le paysage intellectuel. Il écrit à sa femme en juin :

J'ai fait la connaissance de Claudel, mais notre conversation n'a porté ni sur le mysticisme, ni sur le théâtre philosophique et symbolique, mais seulement sur les fabriques de conserves de Naples et sur les listes de " contingentements " Je suis moi-même assez ahuri de l'autorité avec laquelle j'aborde maintenant ces matières. $\mathrm{Au}$ fond, c'est bien plus difficile de faire de la musicologie (Prunières 1916).

Tout en poursuivant ses activités à la mission économique française de Rome ${ }^{9}$, Prunières reste en contact étroit avec Cortot avec lequel il organise des concerts de musique française en Italie et en Suisse.

C'est à cette époque que Prunières développe ses relations avec plusieurs musiciens français et qu'il met en place les bases de son futur réseau international. Pour le moment, il s'agit d'organiser, selon les termes de Cortot, des « concerts français [avec] des représentants les plus qualifiés de nos tendances actuelles » (Cortot 1916a). En fait, Prunières a bien l'intention d'organiser des concerts, mais aussi des conférences et des expositions qui s'intégreront au cadre plus général de la propagande française. De Paris, Cortot le soutient dans ses démarches :

Vous pourriez [...] me dire de quels conférenciers il serait possible de faire coïncider le voyage avec les représentations ou concerts en question ; quels seraient les compositeurs que l'on devrait s'efforcer d'envoyer en Italie pour répondre à la curiosité ou à l'intérêt du public ; quels artistes y auraient particulièrement chance de succès, tant dans le domaine théâtral que dans le domaine musical, également, si quelques expositions de peinture ou d'art français offriraient un élément suffisant pour que nous envisagions la possibilité de les organiser (Cortot 1916b).

Rapidement Prunières est en relation avec le Comte de San Martino, directeur de l'Accademia Santa Cecilia. Ce dernier soutient le développement de la musique

à la France en Italie » (Claudel 1968, p. 355). Au printemps 1915, l'Italie se retire de la Triplice, l'entente entre l'Empire Austro-hongrois, l'Allemagne et l'Italie pour rejoindre les Français, les Anglais et les Russes. On comprend donc que la France établit rapidement des relations diplomatiques et commerciales avec l'Italie qui, à la fin de la Guerre s'est vu promettre de nombreux territoires autrichiens en Europe et Allemand en Afrique et devrait donc jouer un rôle prédominant en méditerranée après la Guerre. À propos de la mission économique de Claudel, voir les travaux d'Emmanuel Boudas (Boudas 2015, p. 140-151).

9 Prunières n'a peut-être pas toujours goûté à son activité de diplomate gestionnaire, mais son beau-père lui écrira une lettre dans laquelle il le conforte quant à l'importance de son travail pour l'avenir de la France : «Tu es toujours très occupé. Je ne sais, mon cher Henry, en quoi consistent exactement tes fonctions. Je pense qu'il s'agit de préparer l'après-guerre en étudiant à fond les conditions des douanes, $\mathrm{du}$ fret, des transports et du change dans tous les grands ports et dans les principales villes industrielles d'Italie, ce que ne font guère nos consuls et moins encore les grands seigneurs que sont la plupart de nos ambassadeurs et de ceux de la Carrière. Tu rends donc au pays un grand service, si j'en juge par mes lectures de Victor Cambon, de Lysès et de tous ceux qui font campagne pour le relèvement de la France, après l'effroyable crise que nous traversons » (Vast 1917). Le beau-père de Prunières a vu juste. Il s'agit pour Claudel de créer une union douanière franco-italienne pour éviter que l'Italie ne tombe à nouveau sous le joug économique de l'Allemagne après la guerre (Soutou 1976, p. 325). 
moderne en Italie et fait de l'Académie le bastion du renouveau musical italien. Giovanni Sgambati, pionnier de ce renouveau, y enseigne le piano jusqu'à sa mort en 1915. Alfredo Casella prend alors le poste de Sgambati et crée la "Società Italiana di Musica Moderna » dont les concerts ont lieu à l'Académie. Prunières, appuyé par Casella, met sur pied un comité italien présidé par le Comte de San Martino pour la diffusion de la musique française. Prunières et Cortot obtiennent au même moment la participation de l'Opéra-Comique qui se déplacera en Italie au début de 1917.

Prunières envisageait probablement moins son action parallèle à ses activités de fonctionnaire comme de la propagande que comme une occasion unique d'une part, de diffuser de la musique française à l'étranger et, d'autre part, de créer des liens artistiques qui permettraient, dans l'avenir, la mise en place d'un réseau international d'échange des œuvres musicales et des artistes.

En 1917, sa femme le rejoint à Rome. Ils emménagent au 19 Corso d'Italia, avec Alfredo Casella comme voisin de palier. La demeure des Prunières devient le lieu de rendez-vous des musiciens et des artistes de passage à Rome ${ }^{10}$. C'est aussi à cette époque que le jeune musicologue fait part publiquement de son projet de revue musicale, comme semble le confirmer le critique musical italien Guido M. Gatti :

[La Revue musicale] naquit pendant une de ces soirées au cours desquelles il rassemblait dans sa maison du Corso d'Italia des musiciens, des peintres, des écrivains et des critiques musicaux [...] Je m'entretins à plusieurs reprises, avec lui et Alfredo Casella, du sujet qui nous tenait à cœur : créer une revue pour appuyer le mouvement musical qui se développait dans tous les pays à ce moment et qui s'annonçait avec l'aspect précis d'un bouleversement fondamental (" Hommage à Henry Prunières » 1952-1953, p. 16).

À ce moment, Prunières avait-il déjà envisagé la reprise de la Revue sIM? Les circonstances de la guerre, la mort d'Écorcheville, la création de la SFM par La Laurencie et le développement de ses relations avec des artistes étrangers le poussent plutôt à concevoir un projet nouveau et très ambitieux. Assurément, Prunières méditait sur ce projet depuis le début de la guerre. D'après le souvenir de Claude Delvincourt, alors pensionnaire à la Villa Médicis en 1917, Prunières « pensait à la création d'une revue musicale de grand style dont il [...] parlait souvent avec une certaine anxiété... Pourrait-il jamais réaliser cet ambitieux projet ?" (ibid., p. 13). Prunières nous en apporte la confirmation dans ce qu'il écrit en 1919 à Romain Rolland :

Je n'ai pas abandonné mon projet d'une grande revue européenne de musique, projet que j'ai étudié et muri durant cinq ans tant au point de vue artistique qu'au point de vue commercial, mais il faudrait de l'argent pour le réaliser et ce n'est pas facile à trouver si l'on entend demeurer libre (Prunières 1919).

10 «Ma femme étant venue me rejoindre avec mes enfants, tous les jeudis soirs, mes amis musiciens se retrouvaient dans notre appartement du Corso d'Italia, près de la via Due Macelli (qu'avait occupé Claudel avant moi). Les artistes de passage s'y retrouvaient avec l'élite de la musique italienne, Malipiero, Casella, Respighi, des poètes, des écrivains, des amateurs » (Lefèvre 1929, p. 1). 
L'anxiété de Prunières tient à ce qu'il sait combien il sera difficile de financer une telle revue sans faire appel aux mécènes, aux associations de défenses de la musique française ou encore aux grands commerçants de la musique qu'étaient alors les éditeurs de musique et les agents d'artistes et qui auraient négocié leur participation moyennant l'appui de la revue à leurs activités, quelle qu'en soit la qualité intrinsèque. Dans cette optique, ces années de gestation ne sont pas inutiles, car lorsque Prunières se lance dans l'aventure, il use de toutes ses relations, la plupart nées de ses activités pendant la guerre, pour créer une revue indépendante des réseaux habituels de la presse musicale.

\section{Le RéSeau de guerre D’Henry Prunières}

Les Prunières rentrent à Paris en juin 1919. Jusqu'à présent, nous n'avons trouvé que très peu de traces de l'activité de Prunières pour les derniers mois de 1919. Mais si l'on se fie à sa correspondance et aux archives d'Henry Le Bœuf ${ }^{11}$, l'un des principaux administrateurs de la future revue, le musicologue met rapidement le pied à l'étrier et se sert de toutes ses relations de guerre pour réaliser le projet de la « Grande revue musicale ». Ce n'est pas tant des collaborateurs qu'il cherche d'abord, mais des investisseurs et des administrateurs. Revoyons ainsi ceux et celles qu'il croise entre 1915 et 1918 et qui joueront un rôle dans la création de La Revue musicale.

C'est probablement Paul Claudel qui met Prunières en relation avec l'équipe de la Nouvelle Revue française (NRF) parmi lesquels il faut mentionner Jacques Rivières dont l'écrivain catholique fut le mentor. C'est par cette voie que Prunières signe une entente avec le directeur des éditions Gallimard dont dépend la NRF, Gaston Gallimard. Les éditions Gallimard fourniront à La Revue musicale les services initiaux de diffusion et de vente.

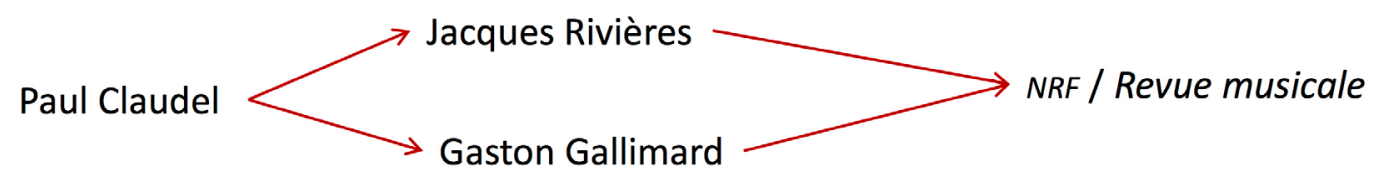

Figure 3: Le premier maillon du réseau de guerre d'Henry Prunières.

Le Comte de San Martino, directeur de l'Academia Santa Cecilia, fera partie de la liste des premiers souscripteurs de la revue et sera membre de son conseil d'administration dans les premières années de fonctionnement de la revue. Des hauts fonctionnaires et des politiciens rencontrés pendant la guerre dans le cadre de ses activités de propagande feront partie des fondateurs de la revue : Georges Noblemaire (1867-1923), administrateur de la Compagnie des chemins de fer de Paris à Lyon et à la Méditerranée, attaché militaire à l'Ambassade de France à Rome pendant la guerre puis député en 1919, sera actionnaire de la revue.

11 À propos de la relation entre Prunières et Henry Le Bœuf, voir Malou Haine (2015) que je remercie pour m'avoir communiqué l'ensemble des documents disponibles dans les archives de l'homme d'affaire. Ces archives sont conservées à la Bibliothèque Royale de Belgique à Bruxelles. 


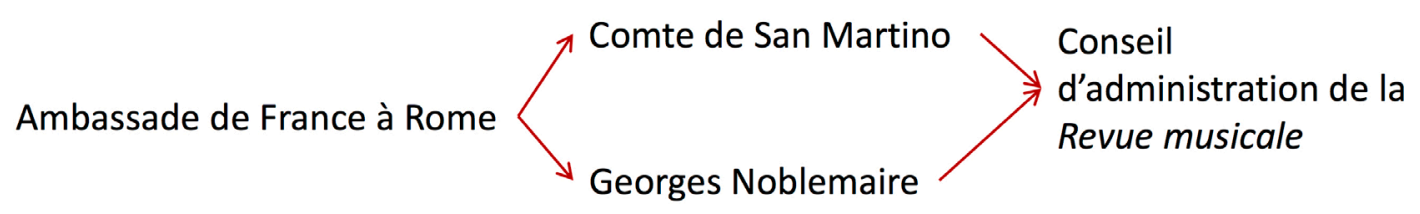

Figure 4: Le deuxième maillon du réseau de guerre d'Henry Prunières.

Parmi les premiers administrateurs de la revue, arrêtons-nous sur le nom d'Henry Le Bœuf, homme d'affaires à la tête de la Banque d'Outremer et mécène belge. Les archives d'Henry Le Bœuf conservent de nombreux documents sur les débuts de la $R M$. La première lettre entre l'homme d'affaires et Prunières date du 10 avril 1920 (Haine 2005, p. 385). Cette lettre est la trace la plus ancienne que nous connaissons de leur relation, mais compte tenu de l'avancement de leurs actions communes pour la constitution de la revue en avril 1920, on imagine sans peine qu'il se connaisse déjà depuis un certain temps. Pendant la guerre, Le Bœuf est responsable du Crédit Foncier d'Extrême-Orient installé à Paris. Comment rencontre-t-il Prunières ? L'hypothèse d'un rapprochement par l'intermédiaire des affaires économiques des Français en Italie pendant la guerre, qui avaient entre autres pour dessein d'établir des liens portuaires pour les ressources en provenance du Proche-Orient et de l'Asie, n'est pas à écarter. La correspondance des deux hommes permet aussi de confirmer que Le Bœuf rétablit les ponts avec l'un des anciens rédacteurs de la SIM pour la Belgique, René Lyr ${ }^{12}$. L'objectif est d'obtenir de la part de Lyr, les listes des abonnés belges de la Revue SIM et toutes les informations susceptibles de favoriser le bon fonctionnement de la nouvelle revue.

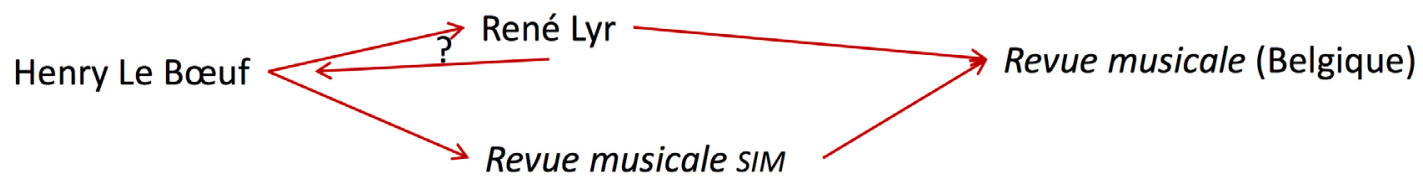

Figure 5: Le troisième maillon du réseau de guerre d'Henry Prunières.

C'est pendant la guerre que Prunières rencontre plusieurs de ses futurs collaborateurs de la revue comme les compositeurs Casella et Malipiero et le critique Guido Gatti qui assurera très régulièrement la chronique musicale en provenance d'Italie; ou encore Cortot qui signera une vingtaine de contributions dans La Revue musicale. En organisant des concerts de musique française tout au long de la guerre, en France puis en Italie, il fréquente un grand nombre de musiciens qui deviendront des ambassadeurs de La Revue musicale et que l'on retrouvera dans le cadre des concerts de La Revue musicale. 

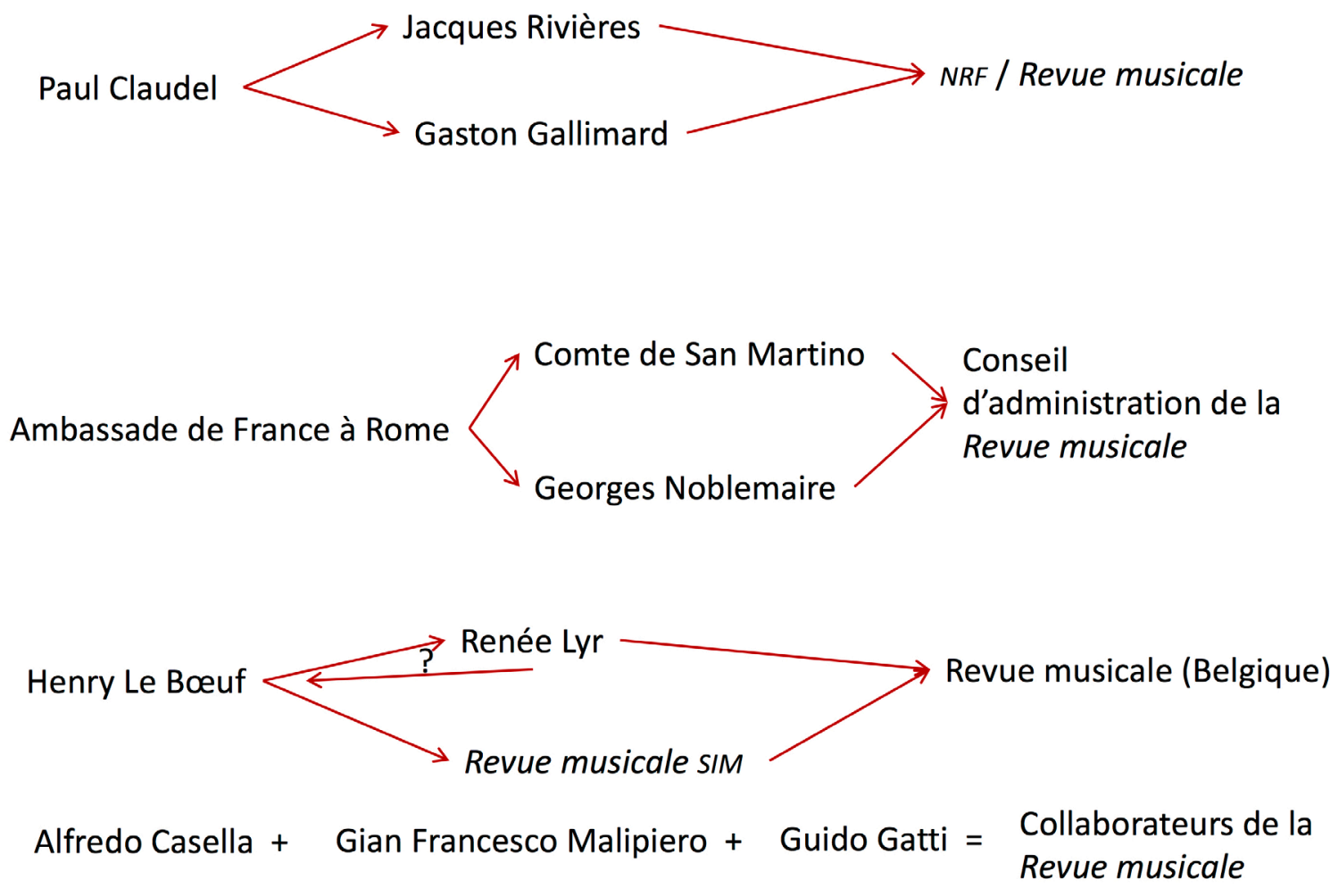

Figure 6: Le réseau de guerre d'Henry Prunières dans sa globalité.

\section{L'APRÈS-GUERRE : DÉPLOIEMENT DU RÉSEAU}

Il est difficile d'extrapoler sur ce qu'aurait pu être l'avenir de La Revue musicale SIM si la guerre n'avait pas eu lieu. Il nous est par contre possible de voir combien l'édifice était fragile et que les dissidences sur fond de nationalisme l'ont rapidement démantelé. Dans un contexte de guerre, où une grande partie de la population est « mobilisée » sur le front ou dans les usines pour soutenir l'effort de guerre, il n'y a plus guère de place dans les esprits pour des revues liées à un milieu culturel aussi spécialisé que celui de la musique. Les ressources réduites ${ }^{13}$ de la presse se concentrent sur les quotidiens et sur une presse de propagande qui occupent toute la sphère médiatique. La disparition de la majorité des titres et la parution sporadique des rares revues musicales survivantes en témoignent. Mais si la guerre a été l'occasion de réduire à néant un projet international comme celui de La Revue musicale SIM, elle permettra à Henry Prunières de concevoir et de réaliser une nouvelle version du projet, sur des bases financières apparemment plus solides, s'écartant des intérêts idéologiques des diverses factions musicales en présence.

13 Le milieu de la presse française souffre, comme tous les autres milieux, d'une hémorragie de main d'œuvre, partie au front. Les typographes, par exemples, ne sont pas exemptés de la mobilisation. Le prix du papier monte en flèche, le pouvoir d'achat des lecteurs diminue (Charle 2004, p. 229-230). La presse, sous toutes ses formes, subit les contrecoups d'une propagande nationalise. On peut ainsi donner l'exemple de la création de la revue La Musique pendant la guerre (voir l'article de Liouba Bouscant dans ce numéro). 
Par contre, Prunières n'abandonne pas l'orientation artistique et littéraire de La Revue musicale SIM qui lui permet d'offrir à la musicologie un cadre ouvert sur un lectorat nettement plus étendu que celui des musicologues français, peu nombreux avant 1914, et encore moins nombreux après la guerre. Compositeurs et littéraires s'exprimeront régulièrement dans la revue qui consacre aussi de nombreuses pages de chacun de ses numéros à des comptes rendus de la vie musicale en France et à l'étranger. Dans les années 1920, avant que la radio ne devienne un moyen efficace de communication, La Revue musicale sert de relais entre monde musical et mélomanes. Le projet de Prunières inscrit donc la musicologie dans une dynamique de diffusion étendue des travaux historiques mais aussi et beaucoup de travaux sur la situation contemporaine de la musique. Prunières est ainsi en accord avec les principes fondamentaux qu'Écorcheville avaient établis avant la guerre pour la Revue SIM. Si la création musicale est souvent au cœur de sa revue, il fera en sorte qu'elle soit par contre le moins souvent possible l'objet de polémiques. Il s'agit évidemment d'éviter de placer la revue dans une situation précaire face à une partie de son lectorat. Mais cette position de relatif consensus a un autre effet. Elle rend le contenu de la revue moins engagé que celui que l'on trouve dans les revues d'avant-guerre qui lui donneront naissance (Mercure musical et Revue SIM). Ce contenu est empreint d'une certaine objectivité qui s'apparente à l'objectivité à laquelle doit se soumettre une science comme la musicologie. Les débats qui auront lieu dans La Revue musicale de Prunières seront menés de manière à éviter toute polarisation institutionnelle, politique et encore moins personnelle ${ }^{14}$. L'équilibre sera fragile, mais Prunières s'évertuera à le maintenir ce dont il témoigne en 1929 dans l'entrevue qu'il accordera à Frédéric Lefèvre dans Les Nouvelles littéraires:

Le devoir du critique musical digne de ce nom me semble être de tenter les plus grands efforts pour dissiper l'ignorance du public et ses préventions absurdes, lui faire aimer à la fois l'art du passé qu'il ne soupçonne pas et l'art du présent qu'il abomine a priori. C'est dans cet esprit que j'ai fondé en 1920 La Revue musicale (Lefèvre 1929, p. 5).

Il ne faut cependant pas oublier le contexte matériel de l'entreprise. Sans ouverture très concrète du milieu universitaire français à la musicologie, il s'agit aussi pour Prunières de construire sa propre entreprise afin de lui permettre de vivre de son métier de musicologue étendu ici aux fonctions d'éditeur. Les frontières entre ses intérêts personnels et ceux de la discipline seront parfois difficiles à identifier et les parutions de La Revue musicale dans l'entre-deux-guerres oscilleront entre la " bonne affaire ", avec certains numéros spéciaux consacrés à des musiciens célèbres, et des numéros d'une grande originalité et au contenu musicologique de tout premier ordre ${ }^{15}$.

14 On pense par exemple aux échanges réfléchis et très argumentés entre Charles Koechlin et Boris de Schloezer à propos du « Retour à Bach » en 1926 et 1927.

15 On peut citer les numéros spéciaux sur la Musique de ballet au XIX siècle (décembre 1921), sur Ronsard et la musique (mai 1924), Géographie musicale (juillet-août 1931) ou encore sur Bach (décembre 1932). 


\section{CONCLUSION}

Prunières construit les assises de sa revue sur l'expérience de la Revue sIM et profite $\mathrm{du}$ fort courant international qui circule dans les milieux artistiques et intellectuels d'Europe à la fin de la guerre. Le destin de la nouvelle revue sera celui d'une grande revue qui aura fait fructifier une expérience d'avant-guerre exceptionnelle, mais dont l'assemblage d'éléments hétéroclites ne résistera pas aux secousses de la Grande Guerre. Au fil des événements, des rencontres, de la constitution d'un réseau économique et intellectuel nouveau, entre 1914 et 1919, Prunières réunira patiemment les pièces nécessaires à la constitution de la plus célèbre revue musicale française de la première moitié du $\mathrm{xx}^{\mathrm{e}}$ siècle : La Revue musicale.

\section{BIBLIOGRAPHIE}

"Avis aux lecteurs" (1907), Le Mercure musical et bulletin de la Société internationale de musique, 15 janvier.

Boudas, Emmanuel (2009), L'Italie et les Alliés de 1914 à 1919. Indépendance ou subordination ?, thèse de doctorat en histoire, Paris, Université de la Sorbonne nouvelle-Paris III.

Charle, Christophe (2004), Le siècle de la presse (1830-1939), Paris, Seuil.

Claudel, Paul (1968), Journal, tome 1 (1904-1932), Paris, Gallimard.

Cortot, Alfred (1916a), lettre à Henry Prunières du 19 juin, Archives privées Henry Prunières (AHP).

Cortot, Alfred (1916b), lettre à Henry Prunières du 26 juin, Archives privées Henry Prunières (AHP).

Duchesneau, Michel (2015), "French Musicology and the Musical Press (1900-1914). The Case of La Revue Musicale, Le Mercure musical and La Revue musicale sIM», Journal of the Royal Musical Association, vol. $140, \mathrm{n}^{\circ} 2$, p. 243-272.

Écorcheville, Jules (1914), lettre à Émile Vuillermoz du 10 septembre, Fonds Jules Écorcheville.

Écorcheville, Jules (1915a), lettre à Madeleine Écorcheville du 18 janvier, Fonds Jules Écorcheville.

Écorcheville, Jules (1915b), lettre à Madeleine Écorcheville du 24 janvier, Fonds Jules Écorcheville.

Direction, La (1914), [Avis aux lecteurs], Le Ménestrel, vol. 80, n 36 (5 septembre), p. 1.

Haine, Malou (2015), « Une amitié franco-belge. Henry Le Bœuf, Henry Prunières et la naissance de La Revue musicale ", dans Myriam Chimènes, Florence Gétreau et Catherine Massip (dir.), Henry Prunières (1886-1942). Un musicologue engagé dans la vie musicale de l'entre-deux-guerres, Paris, Société Française de musicologie, p. 329-403.

« Hommage à Henry Prunières » (1952-1953), brochure de La Revue musicale, p. 1-43.

Iglesias, Sara (2009), " "Le devoir est partout". Reflets de la guerre dans la fondation de la Société française de musicologie en 1917 ", dans Stéphane Audoin-Rouzeau, Esteban Buch, Myriam Chimènes et Georgie Durosoir (dir.), La Grande Guerre des musiciens, Lyon, Symétrie, p. 203-214.

Lefèvre, Frédéric (1929), "Une heure avec M. Henry Prunières », Les Nouvelles littéraires, 5 octobre, p. 1 et 5.

Massip, Catherine (2015), "Romain Rolland, maître, mentor et ami ", dans Myriam Chimènes, Florence Gétreau et Catherine Massip (dir.), Henry Prunières (1886-1942). Un musicologue engagé dans la vie musicale de l'entre-deux-guerres, Paris, Société Française de musicologie, Paris, p. 59-73.

Prunières, Henry (1914a), lettre à Camille Prunières du 26 août, Archives privées Henry Prunières (AHP), 2 lettres.

Prunières, Henry (1914b), lettre à Camille Prunières du 4 septembre, Archives privées Henry Prunières (AHP), 2 lettres. 
Prunières, Henry (1916), lettre à Camille Prunières du 23 juin, Archives privées Henry Prunières (AHP).

Prunières, Henry (1919), lettre à Romain Rolland du 29 mai, Archives privées Henry Prunières (AHP).

Segond-Genovesi, Cédric (2015), «Du Mercure à La Revue musicale (1905-1927). Enjeux et étapes d'une filiation ", dans Myriam Chimènes, Florence Gétreau et Catherine Massip (dir.), Henry Prunières (1886-1942). Un musicologue engagé dans la vie musicale de l'entre-deux-guerres, Paris, Société Française de musicologie, Paris, p. 357-384.

Soutou, Georges (1976), " La politique commerciale et industrielle de la France en Italie pendant la Première Guerre mondiale ", dans La France et l'Italie pendant la Première Guerre mondiale, actes du colloque tenu à l'Université de sciences sociales de Grenoble les 28, 29 et 30 septembre 1973, Grenoble, Presses universitaires de Grenoble.

Vast, Henry (1917), lettre à Henry Prunières du 5 juillet, Archives privées Henry Prunières (AHP).

Vuillermoz, Émile (1915), lettre à Madeleine Écorcheville du 13 décembre, Fonds Jules Écorcheville.

Vuillermoz, Émile (1916), « La Revue sIM », Le Tombeau de Jules Écorcheville, Paris, Dorbon, p. 34. 